\title{
Implicit and Explicit Attitudes Toward Autistic Adults
}

\author{
Cheryl L. Dickter, PhD, Joshua A. Burk, PhD, Janice L. Zeman, PhD, and Sara C. Taylor, BS²
}

\begin{abstract}
Background: Research examining attitudes toward autistic adults has relied on explicit self-report measures, which may be susceptible to socially desirable responding. Because implicit attitudes predict behavioral rejection, understanding both implicit and explicit attitudes toward autistic adults is important. Furthermore, previous research has almost exclusively examined attitudes toward autistic children and has not investigated attitudes toward autistic adults who may also experience prejudice from their peers.

Methods: We created an implicit association test (IAT) to examine implicit attitudes toward autistic adults. In Study 1, we examined 94 neurotypical adults' (mean $[M]_{\text {age }}=31.37$ years) implicit attitudes and explicit attitudes toward autistic adults as well as autistic behaviors. In Study 2 ( $n=137 ; M_{\text {age }}=33.43$ years), we assessed the same variables using an IAT with descriptive rather than stereotypical words.

Results: Participants from both studies demonstrated negative implicit attitudes but positive explicit attitudes toward autistic adults. In Study 2, analyses examining self-reported traits related to autism revealed that more autistic behaviors were associated with less implicit bias.

Conclusions: These findings may help explain why autistic adults report discrimination from their peers. The results suggest that there may be benefits in modifying interventions that reduce implicit bias toward other marginalized groups for use with implicit bias against autistic adults.
\end{abstract}

Keywords: autism, stereotypes, implicit attitudes, explicit attitudes, autistic behaviors

\section{Lay Summary}

Why was this study done?

The goal of this study was to understand how neurotypical adults in the United States feel and think about autistic adults. Negative attitudes can lead to discrimination against autistic adults or to harmful interactions between autistic and neurotypical adults. Although research has previously examined the attitudes that neurotypical adults have toward autistic adults, most of this work has directly asked people about their attitudes, assessing their explicit, or conscious, attitudes. Neurotypical adults, however, may not be able or willing to admit that they have negative attitudes toward autistic adults. Therefore, it is important to evaluate implicit attitudes, which are underlying attitudes at the unconscious level of awareness.

What was the purpose of this study?

This study investigated the implicit and explicit attitudes that neurotypical adults in the general U.S. population have about autistic adults. Assessing both kinds of attitudes is important because each type of attitude predicts different sorts of behaviors toward and judgments of individuals.

\section{What did the researchers do?}

We conducted this study online using a crowdsourcing method of data collection (Amazon's Mechanical Turk) that gave us access to adults throughout the United States. We developed and administered a reaction-time task to examine implicit attitudes toward autistic adults. In this task, participants rapidly categorized words associated with autism and words not associated with autism as being "good" or "bad." We assessed explicit

\footnotetext{
${ }^{1}$ Department of Psychological Sciences, William and Mary, Williamsburg, Virginia, USA.

${ }^{2}$ Departments of Neuroscience and Genetics, University of Pennsylvania, Philadelphia, Pennsylvania, USA.
} 
attitudes with questions about people's knowledge of autism and their liking for autistic adults. Study 1 measured 94 neurotypical adults' (average age $=31.37$ years) implicit and explicit attitudes toward autistic adults; Study 2 measured 137 neurotypical adults' (average age $=33.43$ years) implicit and explicit attitudes. Whereas Study 1's implicit task used words associated with stereotypes about autistic adults (e.g., extraverted, independent), Study 2 used nonstereotypical words associated with autism (e.g., autistic, spectrum).

\section{What were the results of the studies?}

Participants in both studies reported positive explicit attitudes but negative implicit attitudes toward autistic adults. In one study, we also found that neurotypical adults with more autistic traits themselves had more positive implicit attitudes toward autistic adults.

\section{What do these findings add to what was already known?}

Although previous research examined neurotypical adults' explicit attitudes toward autistic adults, the current study demonstrated that neurotypical adults hold negative implicit attitudes toward autistic adults. These findings may help explain why autistic adults experience discrimination from neurotypical adults. Furthermore, our findings suggest that having more autistic traits can lead to a better understanding of the behaviors associated with autism.

\section{What are the potential weaknesses in the study?}

Limitations of the study were that we collected the data online rather than in person and we only included neurotypical adults as participants.

How will these findings help autistic adults now or in the future?

These results shed light on underlying reasons for the potential negative judgments and discrimination that autistic adults face from neurotypical adults. These findings should encourage policy makers to design and implement training programs to reduce neurotypical adults' negative attitudes toward autistic adults.

\section{Implicit and Explicit Attitudes Toward Autistic Adults}

A PPROXIMATELY 1 IN 59 CHILDREN in the United States is diagnosed with autism spectrum disorder. ${ }^{1}$ Autism is associated with social challenges such as communication delays, impaired social skills, and repetitive behaviors that can negatively affect social interactions. ${ }^{2}$ These challenges are amplified by societal barriers such as lack of accommodations and the presence of negative attitudes about autism. ${ }^{3}$ Research regarding attitudes toward this substantial portion of the population is critical to understand ways in which this group may be disadvantaged. The research findings examining attitudes toward autism are inconsistent, which perhaps is not surprising given the strengths and challenges associated with autism. That is, some studies have demonstrated that autistic children and adults are perceived negatively, whereas others suggest that they are perceived positively, and still others find that perceptions vary as a function of the behavior displayed and whether they are identified as autistic. ${ }^{4-9}$ Most studies have relied on a single methodology, namely explicit selfreport measures.

Research has examined attitudes about autistic children and adults. Studies have revealed that autistic children are generally judged negatively by their peers. ${ }^{4,5}$ Health care professionals similarly have negative perceptions about children with developmental disorders such as the perception that they are unintelligent. ${ }^{9}$ Neurotypical adults perceive autistic children more negatively, ascribing traits such as "awkward" to them. ${ }^{7}$ In addition, neurotypical adults and adolescents per- ceive autistic adults more negatively (e.g., more awkward, less likeable) than neurotypical adults, have misconceptions about autism, and are less willing to engage with autistic adults during a first meeting than neurotypical adults. ${ }^{10-17}$ The negative attitudes of neurotypical adults toward autistic adults can lead autistic adults to experience discrimination such as social exclusion, humiliation, and neglect. ${ }^{18-20}$ These experiences may contribute to the high rate of affective disorders in autistic samples, ${ }^{21}$ including depression ${ }^{22}$ and anxiety. ${ }^{23}$ Other studies have found that neurotypical adults hold positive attitudes toward autistic children; two studies, for example, demonstrated that teachers generally have positive attitudes toward autistic children. $^{24,25}$ It is important to note that because both these studies focused on the attitudes of teachers, who have taken special education classes, these findings may not generalize to non-educators. Taken together, these studies have found mixed results and have relied on explicit self-reported attitudes.

Studies that use self-report measures can suffer from social desirability biases; that is, participants may be unwilling to report their true attitudes due to sensitivity about social norms regarding stereotypes. ${ }^{26-28}$ In addition, people can hold negative implicit attitudes that are activated outside of awareness but still influence behavior. ${ }^{29,30}$ Self-reports of attitudes toward autistic adults may thus underestimate these stigmas.

To characterize attitudes toward members of social groups more fully, both self-report and implicit measures need to be included, particularly because each construct predicts different types of behavior. Implicit attitudes predict subtle forms of bias and discrimination such as behavioral rejection or discomfort, ${ }^{31,32}$ whereas explicit attitudes predict 
behaviors that are under conscious control like friendliness toward an individual. ${ }^{33,34}$ Understanding both implicit and explicit attitudes toward stigmatized group members can help predict different forms of discriminatory behavior toward them.

Implicit attitudes are considered to be outside of perceivers' conscious awareness and are typically assessed with measures such as the implicit association test (IAT). ${ }^{35,36}$ The IAT assesses unconscious attitudes about two groups and two attributes (e.g., pleasant, unpleasant). The difference in reaction times (RTs) to trials pairing one group and one attribute (e.g., autistic-pleasant) compared with RTs to trials pairing the group with the other attribute (e.g., autistic-unpleasant) is thought to reflect the strength of the association between the first and the second pair. Disabilities-focused IATs have generally found that participants show an implicit bias against terms related to mental illness. ${ }^{37,38}$ Research has established that implicit and explicit attitudes represent separate but weakly related constructs, with meta-analyses demonstrating small positive correlations of 0.14 and 0.24 between the two types of attitudes. ${ }^{39,40}$ There is only one study examining relationships between explicit and implicit measures toward adults with disabilities that revealed a small but nonsignificant correlation $(r=.17)$ between self-reported explicit attitudes toward autistic adults and implicit attitudes toward adults with disabilities. ${ }^{41}$

For the current research, our first aim was to develop an IAT to examine adults' implicit attitudes toward autistic adults. Based on previous research demonstrating that people exhibit negative implicit attitudes toward those with disabilities, ${ }^{38,42}$ we hypothesized that participants would demonstrate negative implicit attitudes toward autistic adults. Our second aim was to assess the explicit biases that individuals hold of autistic adults using a validated scale of social attitudes. Previous research demonstrates conflicting findings regarding negative and positive perceptions of autism, so we did not have a specific hypothesis regarding explicit bias. Our third aim was to investigate the relation between implicit and explicit attitudes toward autistic adults. We expected that there would either be no correlation or a small positive correlation based on research demonstrating that implicit and explicit measures of attitudes toward disabilities assess different constructs but are related to a small degree.$^{39,40}$ Fourth, we sought to determine whether adults who report more autistic behaviors would show less implicit bias toward autistic adults than those who report fewer autistic behaviors. Previous research indicates that attitudes toward autistic adults tend to be more positive in those individuals with previous experience with autistic people. ${ }^{43}$ Therefore, it is possible that adults with autistic traits may be less biased than those without autistic traits since they may be able to recognize and understand the behaviors in themselves. To address these four aims, we conducted two studies measuring the implicit attitudes, explicit attitudes, and self-reported autistic behaviors of a sample of adults representative of the U.S. national population.

\section{Study 1: Method}

\section{Participants}

Participants were 99 adults (51 men, 48 women; mean $[M]_{\text {age }}=31.30$ years, standard deviation $[S D]=8.05$; range: 19-57 years; 10 East Asian, 10 Black, 6 Hispanic, 67 White, 1 South Asian, 4 multiracial, 1 did not respond) from the United States who participated for payment through Amazon's Mechanical Turk, an online crowdsourcing data collection site that contains a nationally representative sample of consumers. The advantage of using Mechanical Turk (MTurk) for data collection is that it provides easy access to a large, stable, and diverse subject pool. ${ }^{44}$ The data collected from MTurk have been shown to be as reliable as those obtained in the laboratory based on test-retest reliabilities of individual difference measures. ${ }^{45}$ The Protection of Human Subjects Committee from the authors' university approved the study, and participants provided informed consent.

Implicit measures. We developed a modified version of the IAT $^{35}$ using Inquisit software (www.millisecond.com). The IAT measures RT while participants categorize stimulus words into superordinate categories. We conducted two pilot tests to select appropriate stimulus words for adapting the IAT to examine attitudes toward autistic adults. MTurk workers $(n=35)$ generated as many words as they could to describe neurotypical and autistic adults. The words that were most frequently used for one category but infrequently used for the other category ( 37 words) were chosen for a second pilot test in which participants $(n=100)$ indicated the extent to which each word was associated with autistic and nonautistic adults (order counterbalanced between participants) on a 7-point scale from "not at all" to "extremely." We chose words that received the highest values on one category and the lowest values for the other as words for the categories neurotypical (normal, extroverted, functional, typical, independent, social) and autistic (different, challenged, special, dependent, impaired, disabled). The words associated with pleasant and unpleasant categories were the standard IAT words (i.e., marvelous, superb, pleasure, joyful, beautiful, glorious, horrible, awful, tragic, agony, painful, terrible). Before participants began the task, they were shown the words that were associated with each category.

All stimulus words appeared in the middle of the screen with the category words presented in the top right and top left portions of the screen, as depicted in Figure 1. As specified by Greenwald et al., ${ }^{35}$ the first two blocks consisted of 20 trials each, with participants categorizing autistic and neurotypical words with one response key on a keyboard, and "good" and
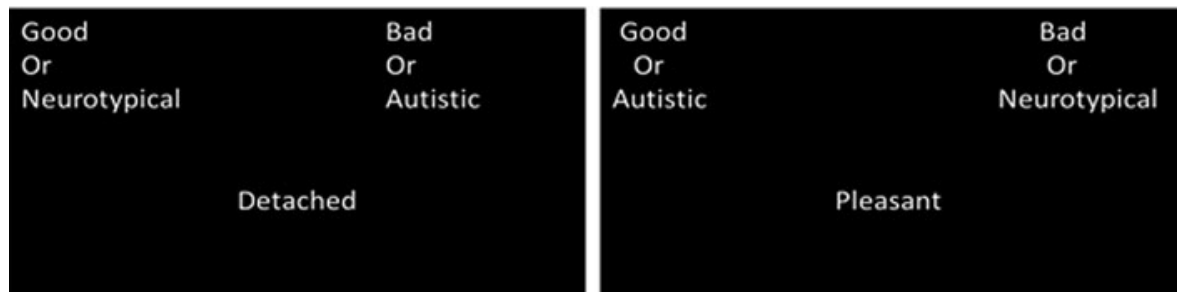

FIG. 1. Visual representation of IAT task. IAT, implicit association test. 
"bad" words with another key. In Blocks 3 and 4, participants grouped all words into one of two categories, using a response key designated for each: half of the participants were randomly assigned to have "good/neurotypical" and "bad/autistic" as the two response options and half were randomly assigned to use bad/neurotypical and good/autistic as the response options. Block 3 had 20 trials and Block 4 had 40 trials. In Block 5, the response keys for "bad" and "good" changed and participants completed 40 trials with this new association. Two final blocks had participants group all words into the other pairing of categories: Block 6 had 20 trials and Block 7 had 40 trials. For each trial, the words appeared until participants responded. If they responded incorrectly, a red " $\mathrm{X}$ " appeared on the screen until they made the correct response. After participants made a correct response, there was a $250 \mathrm{~ms}$ intertrial interval. We counterbalanced the first presentation of the "good/autistic" or "bad/autistic" blocks.

Explicit measures. The Societal Attitudes Towards Autism Scale S1 $^{4}$ (SATA) is a 16-item inventory to which participants respond using a 4-point scale $(1=$ strongly disagree to $4=$ strongly agree). The SATA contains items such as "People with autism should not engage in romantic relationships." Validity and reliability have been established in a neurotypical sample of adults. ${ }^{38}$ Internal consistency was acceptable $(\alpha=.89)$.

The Autism Quotient $\left(\mathrm{AQ}^{46}\right)$ is a 50 -item inventory in which participants respond to statements about autistic behaviors using a 4-point scale $(1=$ definitely agree to $4=$ definitely disagree). The AQ contains items such as "I tend to have very strong interests, which I get upset about if I can't pursue."' Items are scored dichotomously with a score of 32 or higher suggesting behavior consistent with autism; one score in the current sample was greater than 32. Reliability and validity have been established with samples of autistic and neurotypical adults. ${ }^{46}$ Internal consistency in the present study was acceptable $(\alpha=.76)$.

\section{Procedure}

We recruited participants through MTurk and participants completed all measures online. They first filled out an informed consent using Qualtrics software. Participants then completed the IAT, using Inquisit software, and then a Qualtrics survey with the SATA and AQ items. We included two additional items asking participants to select a specific answer in each questionnaire to ensure that participants were attending to the questions and to prevent the chance that data were infiltrated by bots.

\section{Results}

Participants were excluded if they indicated that they were autistic $(n=3)$ or if they did not complete all parts of the study $(n=13)$. Therefore, data analyses were conducted for 83 participants ( 40 men, 43 women; $M_{\text {age }}=31.37$ years, $S D_{\text {age }}=8.49$ ). There were no gender differences on any of the measures so the analyses below do not include gender.

The IAT was scored using Greenwald et al.'s ${ }^{47}$ revised method, which involves deleting trials with RTs greater than $10,000 \mathrm{~ms}$ and excluding participants for whom more than $10 \%$ of trials had RTs shorter than $300 \mathrm{~ms}$. We calculated two
SDs, one for the trials in Blocks 3 and 6 and another for trials in Blocks 4 and 7. We computed the mean latency for Blocks $3,4,6$, and 7 and then computed the mean differences for Block 6 minus Block 3 and then Block 7 minus Block 4. We then divided each of these difference scores by their respective SDs; the IAT $D$-score represents the average of these two ratios. Higher IAT $D$-scores indicated more negative associations with autism. The mean calculated IAT $D$-score was $0.61(S D=0.48$, range: -1.21 to 1.35$)$, which significantly differed from $0, t(82)=15.50, p<.001$, Cohen's $d=1.34$, indicating an overall implicit bias against autistic adults. Scores on the SATA ranged from 34.00 to 64.00 $(M=51.32, S D=7.86)$. Scores on the AQ ranged from 6.00 to $38.00(M=20.19, S D=6.96)$. Each variable was normally distributed.

We calculated the Pearson correlations to test our third and fourth hypotheses. As depicted in Figure 2A, the correlation between the IAT and the SATA was $r=.20, p=.08$. The IAT score was significantly negatively correlated with the AQ, $r=-.45, p<.001$, demonstrating that participants with higher levels of autistic behaviors showed less implicit bias (Fig. 2B).

\section{Discussion}

One of the limitations of Study 1 was that the words that participants from the pilot test generated were positive and negative stereotypes of autistic adults. Therefore, for Study 2 , we used words that were less stereotypical and more descriptive of both groups of adults (e.g., autistic: spectrum, ASD; nonautistic: typical, nonautistic). In this way, we were
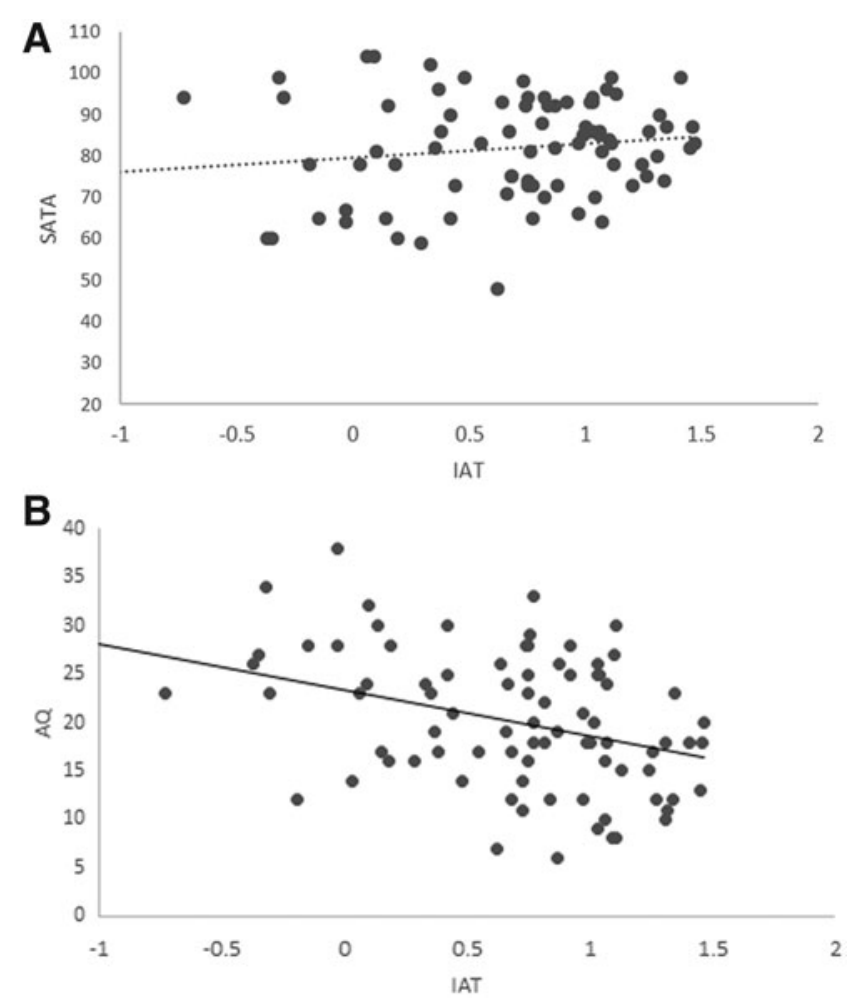

FIG. 2. (A) Scatterplot for the SATA and IAT in Study 1. (B) Scatterplot for the AQ and IAT in Study 1. AQ, Autism Quotient; SATA, Societal Attitudes Towards Autism Scale. 
not priming participants with stereotypes of the target group. Using these new stimuli, we expected to replicate the findings from Study 1.

\section{Study 2: Method}

\section{Participants}

Participants were 135 adults $(77$ men, 57 women, 1 transgender; $M_{\text {age }}=33.64$ years, $S D=9.31$; range: $20-68$ years; 8 East Asian, 16 Black, 7 Hispanic, 90 White, 10 multiracial, 3 did not respond, 1 "other") from the United States who participated for payment through MTurk. The Protection of Human Subjects Committee approved the study, and participants provided informed consent.

\section{Materials}

Experts in the field of autism research viewed the words from the pilot test used in Study 1 and selected words to describe autistic (autistic, spectrum, ASD, Asperger's) and nonautistic adults (typical, nonautistic, usual, non-ASD) that were descriptive rather than stereotypical. We presented the participants with the list of words identifying which words were associated with each category before the task. The IAT was otherwise identical to that of Study 1, as were the items on the SATA $(\alpha=.90)$ and the AQ $(\alpha=.80)$.

\section{Procedure}

We recruited our participants through MTurk; the participants completed all measures online. They first accessed a survey using Qualtrics software and completed the informed consent. They next completed the IAT, which was administered using Inquisit software, and then, they answered the SATA and AQ questionnaires. As in Study 1, we included two attention check questions.

\section{Results}

We excluded participants if they indicated that they were autistic $(n=2)$ or if they did not complete all materials in the study $(n=6)$. Therefore, data analyses were conducted for 127 participants (75 men, 51 women, 1 transgender; $M_{\text {age }}=$ 33.43 years, $S D_{\text {age }}=9.20$ ). There were no gender differences on any of the measures.

We scored the IAT using the same procedure as in Study 1. The mean calculated IAT $D$-score was $0.37(S D=0.49$, range: -0.95 to 1.45$)$, which significantly differed from 0 , $t(127)=8.68, p<.001$, indicating an overall implicit bias against autistic adults. Scores on the SATA ranged from 35.00 to $68.00(M=57.27, S D=7.86)$. Scores on the AQ ranged from 5.00 to $39.00(M=19.77, S D=7.22)$. Each variable was normally distributed.

As depicted in Figure 3A, the results revealed that the IAT score was not significantly correlated with the SATA, $r=.07, p=.456$. Although participants with higher levels of autistic behaviors showed less implicit bias, the correlation between the IAT scores and the AQ did not reach conventional levels of significance, $r=-.15, p=.088$ (Fig. 3B).

\section{General Discussion}

The overarching goal of this research was to increase our understanding of the implicit and explicit attitudes that adults
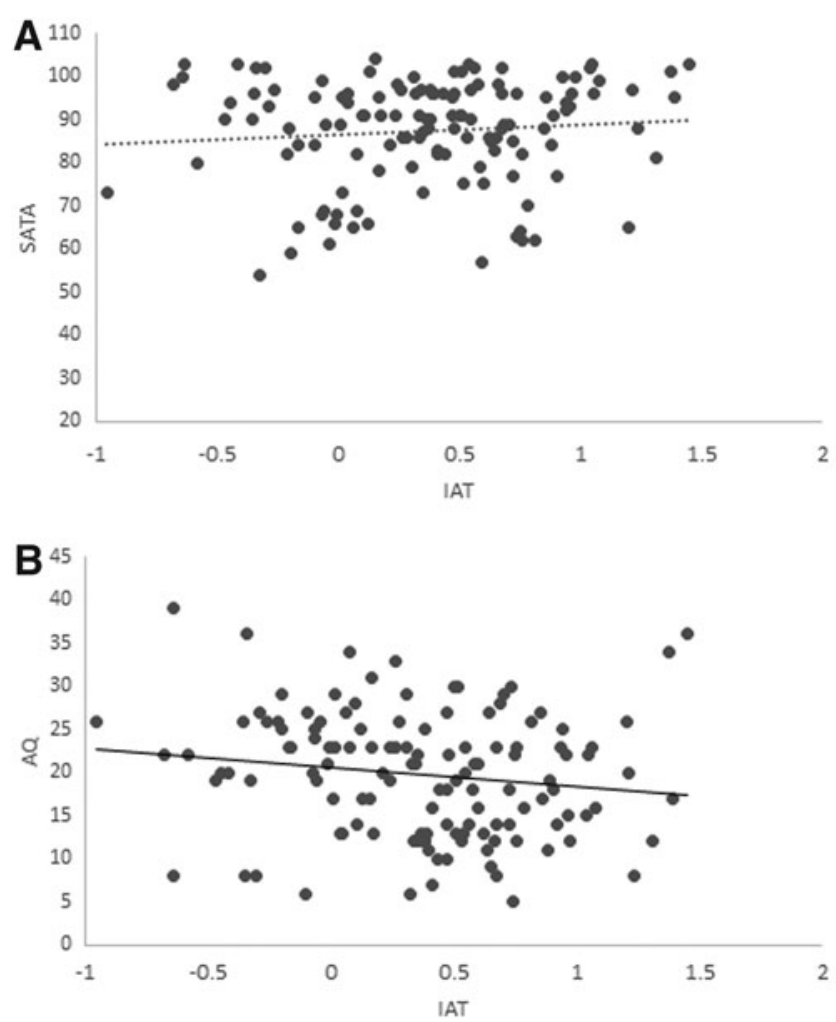

FIG. 3. (A) Scatterplot for the SATA and IAT in Study 2. (B) Scatterplot for the AQ and IAT in Study 2.

in the general U.S. neurotypical population have toward autistic adults. A related goal was to develop an IAT that can evaluate implicit attitudes toward autistic adults. The results of our two studies indicated that the participants showed a negative implicit bias against autistic adults regardless of whether the words used in the IAT were stereotypical or descriptive. These findings are important, as negative implicit attitudes may influence neurotypical people's behavior toward autistic adults, adding to the challenges faced by these individuals. Furthermore, the participants reported positive explicit attitudes that were consistent with those of experts who work with autistic children and adults, as reported in Flood et al. ${ }^{41}$ This research also demonstrated that, in Study 1 , the fewer autistic behaviors participants reported, the more negative their implicit bias was against autistic adults. Given these findings, it is important to consider ways to alter these biases to provide a more accepting environment for autistic adults.

The current studies deepen our understanding of the implicit and explicit attitudes that neurotypical people have regarding autistic adults. Social psychological research has demonstrated that these different methods are predictive of specific types of behavior, with implicit attitudes predicting more subtle forms of bias and discrimination and explicit attitudes predicting more overt behaviors. ${ }^{31-34}$ Each method thus likely predicts different types of behaviors toward autistic adults. The IAT has been shown to have greater predictive validity than explicit measures, ${ }^{47}$ suggesting that the autism IAT may be a better indicator of actual behavior toward autistic adults than the explicit attitude questionnaire. Furthermore, the IAT is a more robust predictor of less controllable biased behaviors such as 
behavioral rejection or feelings of discomfort toward individuals of a disadvantaged group in comparison to explicit measures. $^{32,48}$ Determining whether the implicit and/or explicit attitudes that individuals hold about autism results in discriminatory behavior is an important area for future research. As such, one of the strengths of this set of studies is the creation of a new implicit measure of attitudes toward autistic adults.

Interestingly, the explicit attitudes were positive, consistent with the explicit attitudes displayed by experts in a study by Flood et al. ${ }^{41}$ than with the nonexpert young adults (i.e., college students) in their study. It is unclear whether these positive attitudes are the result of more exposure to and knowledge of autism given its recent depiction in TV shows and the media in general, or whether these attitudes are particular to the samples obtained herein. Future research should explore attitudes toward autism in samples from other cultures and in other age groups.

In the current studies, the relationship between implicit and explicit attitudes toward autistic adults was small and not statistically significant in either Study 1 or Study 2. This is consistent with previous work with social groups (e.g., race), which typically finds small or nonsignificant correlations between implicit and self-report measures, although there is much variability among studies. ${ }^{39,40}$ Our findings are also consistent with the research by Flood et al. ${ }^{41}$ that found a small $(r=.21)$ but nonsignificant correlation between the SATA and the disabilities IAT. Together with this previous work, our findings suggest that these two assessments measure separate constructs.

Importantly, despite making changes to the IAT in Study 2 by using descriptive words associated with autism as opposed to stereotypes in Study 1, there was still a significant negative attitude toward autistic adults. Thus, the neurotypical adults in the current study had negative associations about autistic adults regardless of the stimulus words used in the task. This replication of the findings provides more confidence in the validity and replicability of the results in our studies and also in the IAT developed for this research.

Regarding the relationship between implicit attitudes toward autistic adults and autistic behaviors, in support of our hypothesis, we found that self-report of more autistic behaviors related to less implicit bias, although this relationship did not reach statistical significance in Study 2. Having more autistic traits may be related to a more positive association with autism because these individuals may have a better understanding of the behaviors associated with autism and, as a result of this better understanding, form a more positive association with autism.

Although this research is a novel addition to the literature, its limitations are an impetus for future research directions. First, participants completed the studies in their own environments over which we had no control. Previous research, however, demonstrates that MTurk data are as reliable as those obtained in the laboratory. ${ }^{44}$ Future research may also aim to better characterize the history of interactions participants have had with autistic adults as individuals who have close relationships with autistic adults may show less bias toward this group. ${ }^{25,43}$ Another limitation of the current work is that some individuals may have participated in both studies, although this is unlikely given that all participants completed the session from a different IP address. It is also imperative in future studies to examine whether implicit and explicit attitudes toward autistic adults predict important real-world behavior toward autistic adults such as evaluations related to employment or job performance as well as in colleges and universities. Neurotypical individuals who have more negative attitudes toward autistic adults may evaluate autistic adults more negatively; to our knowledge, research has not yet examined this possibility. It is also important to note that we only included self-identified neurotypical adults in this research, and as such, the range of the AQ scores was likely truncated. Future research could include autistic adults in the sample to provide a greater statistical range that could modify the associations found between the IAT and the AQ in this study. Finally, it may be helpful to collect data regarding what prototype, if any, people generate with regard to autism. That is, discrepant results may arise if one participant thinks of an autistic adult as someone who requires considerable caregiving versus another participant who has experience with an autistic adult who has exceptionalities along with challenges and functions independently and adaptively.

\section{Conclusions}

Together, these studies suggest that neurotypical adults hold negative implicit attitudes toward autistic adults. These findings may help explain why autistic adults report experiences of discrimination such as social exclusion, humiliation, and neglect. The findings reported from the current studies suggest that workplaces and other organizations may need to implement interventions to reduce implicit bias, such as those identified in the implicit race bias literature. ${ }^{49}$

\section{Acknowledgments}

We thank Julie Kittel for her help in organizing the data for Study 1. All procedures performed in studies involving human participants were in accordance with the ethical standards of the institutional and/or national research committee and with the 1964 Declaration of Helsinki and its later amendments or comparable ethical standards. We obtained informed consent from all individual participants involved in each study.

\section{Authorship Confirmation Statement}

Dr. C.L.D. helped design the study, oversaw programming and data collection, analyzed the results, and helped prepare the article. Dr. J.A.B. helped design the study, oversaw data collection, and helped prepare the article. Dr. J.L.Z. helped design the study and helped prepare the article. Ms. S.C.T. helped with data collection and analysis. All co-authors have reviewed and approved the article before submission. This article has been submitted solely to this journal and is not published, in press, or submitted elsewhere.

\section{Author Disclosure Statement}

No competing financial interests exist.

\section{Funding Information}

This research did not receive any specific grant from funding agencies in the public, commercial, or not-for-profit sectors. 


\section{References}

1. Baio J, Wiggins L, Christensen DL, et al. Prevalence of autism spectrum disorder among children aged 8 YearsAutism and Developmental Disabilities Monitoring Network, 11 Sites, United States, 2014. MMWR Surveill Summ. 2018;67(No. SS-6):1-23.

2. American Psychiatric Association. Diagnostic and Statistical Manual of Mental Disorders (5th ed.). Washington, DC: 2013.

3. Oliver M. Politics of Disablement. Hampshire, England: Macmillan International Higher Education; 1990 Aug 1.

4. Campbell JM, Ferguson JE, Herzinger CV, Jackson JN, Marino CA. Combined descriptive and explanatory information improves peers' perceptions of autism. Res Dev Disabil. 2004;25(4):321-339.

5. Swaim KF, Morgan SB. Children's attitudes and behavioral intentions toward a peer with autistic behaviors: Does a brief educational intervention have an effect? J Autism Dev Disord. 2001;31(2):195-205.

6. Chambres P, Auxiette C, Vansingle C, Gil S. Adult attitudes toward behaviors of a six-year-old boy with autism. J Autism Dev Disord. 2008;38(7):1320-1327.

7. Grossman RB. Judgments of social awkwardness from brief exposure to children with and without high-functioning autism. Autism. 2015;19(5):580-587.

8. Mor N, Berkson G. Attitudes toward stereotyped behaviors. J Dev Phys Disabil. 2003;15(4):351-365.

9. Spaniol L, Gagne C, Koehler M. (2003). The recovery framework in rehabilitation and mental health. In: Moxley DP, Finch JR, eds. Sourcebook of Rehabilitation and Mental Health Practice. New York, NY: Springer; 2003;37-50.

10. Cage E, Di Monaco J, Newell V. Understanding, attitudes and dehumanisation towards autistic people. Autism. 2019; 23(6):1373-1383.

11. DeBrabander KM, Morrison KE, Jones DR, Faso DJ, Chmielewski M, Sasson NJ. Do first impressions of autistic adults differ between autistic and nonautistic observers? Autism Adulthood. 2019;4:250-257.

12. Gillespie-Lynch K, Brooks PJ, Someki F, et al. Changing college students' conceptions of autism: An online training to increase knowledge and decrease stigma. J Autism Dev Disord. 2015;45(8):2553-2566.

13. Morrison KE, DeBrabander KM, Faso DJ, Sasson NJ. Variability in first impressions of autistic adults made by neurotypical raters is driven more by characteristics of the rater than by characteristics of autistic adults. Autism. 2019; 23(7):1817-1829.

14. Sasson NJ, Morrison KE. First impressions of adults with autism improve with diagnostic disclosure and increased autism knowledge of peers. Autism. 2019;23(1):50-59.

15. Sasson NJ, Faso DJ, Nugent J, Lovell S, Kennedy DP, Grossman RB. Neurotypical peers are less willing to interact with those with autism based on thin slice judgments. Sci Rep. 2017;7:40700.

16. Sasson NJ, Morrison KE, Pinkham AE, Faso DJ, Chmielewski M. Brief report: Adults with autism are less accurate at predicting how their personality traits are evaluated by unfamiliar observers. J Autism Dev Disord. 2018;48(6):2243-2248.

17. Stevenson JL, Mowad TG. Explicit associations with autism and disability. Autism Adulthood. 2019;1:258-267.

18. Attwood A. Asperger's Syndrome: A Guide for Parents and Professionals. Philadelphia, PA: Jessica Kingsley Publishers; 1998.
19. Gray DE. Negotiating autism: Relations between parents and treatment staff. Soc Sci Med. 1993;36(8):1037-1046.

20. Peyton S. Stigma in our schools and communities. Massachusetts Association of Special Education Parent Advisory Councils. (Available at: www.Hateamongstus.net/ FWH/Comp/StigmainourSchools\&Communiti.Pdf). 2004.

21. Tantam D. Psychological disorder in adolescents and adults with Asperger syndrome. Autism. 2000;4(1):47-62.

22. Hedley D, Young R. Social comparison processes and depressive symptoms in children and adolescents with Asperger syndrome. Autism. 2006;10(2):139-153.

23. White SW, Ollendick TH, Bray BC. College students on the autism spectrum: Prevalence and associated problems. Autism. 2011;15(6):683-701.

24. Olley JG, DeVellis RF, DeVellis BM, Wall AJ, Long CE. The autism attitude scale for teachers. Except Child. 1981; 47(5):371-372.

25. Park M, Chitiyo M. An examination of teacher attitudes towards children with autism. J Res Spec Educ Needs. 2011;11(1):70-78.

26. Dovidio JF, Gaertner SL. Aversive racism and selection decisions: 1989 and 1999. Psychol Sci. 2000;11(4):315-319.

27. Hergenrather K, Rhodes S. Exploring undergraduate student attitudes toward persons with disabilities application of the disability social relationship scale. Rehabil Couns Bull. 2007;50(2):66-75.

28. Pruett SR, Chan F. The development and psychometric validation of the disability attitude implicit association test. Rehabil Psychol. 2006;51(3):202-213.

29. Devine PG, Monteith MJ. Automaticity and control in stereotyping. In: Chaiken S, Trope Y, eds. Dual-process theories in social psychology. New York, NY: Guildford Press; 1999;339-360.

30. Payne BK. Prejudice and perception: The role of automatic and controlled processes in misperceiving a weapon. J Pers Soc Psychol. 2001;81(2):181-192.

31. Dovidio JF, Kawakami K, Gaertner SL. Implicit and explicit prejudice and interracial interaction. J Pers Soc Psychol. 2002;82(1):62-68.

32. Fazio R, Jackson J, Dunton B, Williams C. Variability in automatic activation as an unobtrusive measure of racial attitudes: A bona fide pipeline? J Pers Soc Psychol. 1995; 69(6):1013-1027.

33. Karpinski A, Steinman RB, Hilton JL. Attitude importance as a moderator of the relationship between implicit and explicit attitude measures. Pers Soc Psychol Bull. 2005; 31(7):949-962.

34. Shelton JN, Richeson JA, Salvatore J, Trawalter S. Ironic effects of racial bias during interracial interactions. Psychol Sci. 2005;16(5):397-402.

35. Greenwald AG, McGhee DE, Schwartz JL. Measuring individual differences in implicit cognition: The implicit association test. J Pers Soc Psychol. 1998;74(6):14641480 .

36. Nosek BA, Banaji M, Greenwald AG. Harvesting implicit group attitudes and beliefs from a demonstration web site. Group Dyn Theor Res. 2002;6(1):101-115.

37. Stier A, Hinshaw SP. Explicit and implicit stigma against individuals with mental illness. Aust Psychol. 2007;42(2): 106-117.

38. Thomas A, Doyle A, Daly V. Implementation of a computer based implicit association test as a measure of attitudes toward individuals with disabilities. J Rehabil. 2007; 73(2):3-14. 
39. Hofmann W, Gawronski B, Gschwendner T, Le H, Schmitt M. A meta-analysis on the correlation between the Implicit Association Test and explicit self-report measures. Pers Soc Psychol Bull. 2005;31(10):1369-1385.

40. Oswald FL, Mitchell G, Blanton H, Jaccard J, Tetlock PE. Predicting ethnic and racial discrimination: A metaanalysis of IAT criterion studies. J Pers Soc Psychol. 2013; 105(2):171-192.

41. Flood LN, Bulgrin A, Morgan BL. Piecing together the puzzle: Development of the societal attitudes towards autism (SATA) scale. J Res Spec Educ Needs. 2013;13(2):121-128.

42. Vaughn ED, Thomas A, Doyle AL. The multiple disability implicit association test psychometric analysis of a multiple administration IAT measure. Rehabil Couns Bull. 2011; 54(4):223-235.

43. Nevill RE, White SW. College students' openness toward autism spectrum disorders: Improving peer acceptance. J Autism Dev Disord. 2011;41(12):1619-1628.

44. Mason W, Suri S. Conducting behavioral research on Amazon's Mechanical Turk. Behav Res Methods. 2012; 44(1):1-23.

45. Buhrmester M, Kwang T, Gosling SD. Amazon's Mechanical Turk: A new source of inexpensive, yet high-quality, data? Perspect Psychol Sci. 2011;6(1):3-5.
46. Baron-Cohen S, Wheelwright S, Skinner R, Martin J, Clubley E. The Autism-Spectrum Quotient (AQ): Evidence from Asperger syndrome/high-functioning autism, males and females, scientists and mathematicians. J Autism Dev Disord. 2001;31(1):5-17.

47. Greenwald AG, Nosek BA, Banaji MR. Understanding and using the implicit association test: I. An improved scoring algorithm. J Pers Soc Psychol. 2003;85(2):197-216.

48. Teachman BA, Wilson JG, Komarovskaya I. Implicit and explicit stigma of mental illness in diagnosed and healthy samples. J Social Clin Psychol. 2006;25(1):75-95.

49. Lai CK, Marini M, Lehr SA, et al. Reducing implicit racial preferences: I. A comparative investigation of 17 interventions. J Exp Psychol Gen. 2014;143(4):1765-1785.

Address correspondence to:

Cheryl L. Dickter, PhD

Department of Psychological Sciences

William and Mary

P.O. Box 8795

Williamsburg, VA 23187

USA

Email: cldickter@wm.edu 